PROCEEDINGS OF THE

AMERICAN MATHEMATICAL SOCIETY

Volume 133, Number 1, Pages 245-255

S 0002-9939(04)07605-1

Article electronically published on August 4, 2004

\title{
HERMITE-BIEHLER FUNCTIONS WITH ZEROS CLOSE TO THE IMAGINARY AXIS
}

\author{
MICHAEL KALTENBÄCK AND HARALD WORACEK
}

(Communicated by Joseph A. Ball)

\begin{abstract}
A Hermite-Biehler function $E$ gives rise to a de Branges Hilbert space $\mathcal{H}(E)$ consisting of entire functions. We are going to show that for Hermite-Biehler functions of sufficiently small growth and a certain distribution of zeros every proper de Branges subspace of $\mathcal{H}(E)$ coincides for some $n \in \mathbb{N}$ with the $(n+1)$-dimensional linear space of all polynomials of degree at most $n$
\end{abstract}

\section{INTRODUCTION}

An entire function $E$ is said to belong to the Hermite-Biehler class, $E \in \mathcal{H} B$, if it has no zeros in the open upper half-plane $\mathbb{C}^{+}$and if

$$
\left|E^{\#}(z)\right| \leq|E(z)|, \quad z \in \mathbb{C}^{+},
$$

where we have $F^{\#}(z):=\overline{F(\bar{z})}$ whenever $F$ is an entire function. With a function $E \in \mathcal{H} B$ we associate the space $\mathcal{H}(E)$ of all entire functions $F$ such that

$$
\frac{F}{E}, \frac{F^{\#}}{E} \in H^{2}\left(\mathbb{C}^{+}\right) \text {. }
$$

Here $H^{2}\left(\mathbb{C}^{+}\right)$denotes the Hardy space of the upper half-plane (cf. [7]). If $\mathcal{H}(E)$ is endowed with the norm

$$
\|F\|_{\mathcal{H}(E)}^{2}:=\int_{-\infty}^{\infty}\left|\frac{F(t)}{E(t)}\right|^{2} d t
$$

it becomes a Hilbert space, the so-called de Branges space associated with E. For a detailed discussion of the relationship between de Branges spaces and HermiteBiehler functions we refer the reader to [2], 4].

If $F$ is a function analytic at a point $w$, we denote by $\operatorname{Ord}_{w} F$ the order of $w$ as a zero of $F$. For a de Branges space $\mathcal{H}(E)$, let us define

$$
\mathfrak{d}(\mathcal{H}(E)):\left\{\begin{array}{rll}
\mathbb{C} & \rightarrow \mathbb{N} \cup\{0\}, \\
w & \mapsto \min _{F \in \mathcal{H}(E) \backslash\{0\}} \operatorname{Ord}_{w} F .
\end{array}\right.
$$

A subspace $\tilde{\mathcal{H}}$ of a de Branges space $\mathcal{H}(E)$ is called a de Branges subspace if it is itself, with the norm inherited from $\mathcal{H}(E)$, a de Branges space, i.e. if for some $\tilde{E} \in \mathcal{H} B$ we have $\tilde{\mathcal{H}}=\mathcal{H}(\tilde{E})$ as sets and also $(F, G)_{\mathcal{H}(E)}=(F, G)_{\mathcal{H}(\tilde{E})}$ whenever $F, G \in \tilde{\mathcal{H}}$.

Received by the editors March 15, 2003 and, in revised form, October 7, 2003.

2000 Mathematics Subject Classification. Primary 46E20, 46E22; Secondary 30H05, 30D15. 
It is a deep result of L. de Branges that the set of all de Branges subspaces $\tilde{\mathcal{H}}$ of a given space $\mathcal{H}(E)$, which additionally satisfy $\mathfrak{d}(\tilde{\mathcal{H}})=\mathfrak{d}(\mathcal{H}(E))$, is totally ordered with respect to inclusion. In the sequel we shall refer to this set as the chain of subspaces of $\mathcal{H}(E)$. In [2, Theorem 35] in fact a stronger result is proved dealing with de Branges spaces contained in a space $L^{2}(d \mu)$. The total ordering of the de Branges subspaces of a given space $\mathcal{H}(E)$ which we refer to, is deduced from this by taking $d \mu:=|E(t)|^{-2} d t$. It is apparent from the theory developed in [2] that a lot of information about the structure of the space $\mathcal{H}(E)$ is contained in the structure of its chain of subspaces.

The aim of this note is to determine explicitly the chain of subspaces of $\mathcal{H}(E)$ for a certain subclass of Hermite-Biehler functions $E$, cf. Theorem 2.1. Corollary 2.7, in other words to give a structure result on certain types of de Branges spaces. Roughly speaking we will prove that if $E$ is of reasonably small growth and has a certain distribution of zeros, then the chain of subspaces of $\mathcal{H}(E)$ is order-isomorphic to $\mathbb{N} \cup\{+\infty\}$. This result contains a theorem of [3] as a particular case; cf. Remark 2.3 .

It is interesting to note that examples of Hermite-Biehler functions satisfying our hypothesis come up in number theory. We will in fact show that from even entire functions subject to appropriate growth conditions whose zeros are all located in a strip one can construct examples of Hermite-Biehler functions of the studied kind. In particular, this construction can be applied to the function

$$
\Xi(z):=-\frac{1}{2}\left(z^{2}+\frac{1}{4}\right) \pi^{-\frac{1}{4}-\frac{i z}{2}} \Gamma\left(\frac{1}{4}+\frac{i z}{2}\right) \zeta\left(\frac{1}{2}+i z\right),
$$

which has been intensively studied in the classical theory of the Riemann zeta function.

\section{The structure of $\mathcal{H}(E)$}

Before we come to the statement of our main result let us recall that by 5 . Lemma 3.12] a function $E \in \mathcal{H} B$ that is of finite order with zeros $z_{n}$ that, counted according to their multiplicities, meet the constraint

$$
\sum_{z_{n} \notin \mathbb{R}} \frac{1}{\left|z_{n}\right|}<\infty
$$

can be represented as

$$
E(z)=D(z) e^{i \frac{\tau_{E}}{2} z} \prod_{z_{n} \notin \mathbb{R}}\left(1-\frac{z}{z_{n}}\right)
$$

with an appropriate real function $D$. Thereby $\tau_{E}$ is the so-called mean type of the function $E^{-1} E^{\#}$, i.e.

$$
\tau_{E}=\operatorname{mt} E^{-1} E^{\#}=\limsup _{y \rightarrow+\infty} \frac{1}{y} \log \left|\frac{E^{\#}(i y)}{E(i y)}\right| \leq 0 .
$$

Moreover, we know from [5] Lemma 2.2] that for every function $E \in \mathcal{H} B$ whose nonreal zeros lie in an angle off the real axis actually (2.1) holds.

Recall e.g. from [2] that a space $\mathcal{H}(E)$ is a reproducing kernel Hilbert space. Its reproducing kernel $K(w,$.$) is given explicitly by$

$$
K(w, z)=\frac{E(z) E^{\#}(\bar{w})-E(\bar{w}) E^{\#}(z)}{2 \pi i(\bar{w}-z)} .
$$


Denote by $\mathbb{C}[z]$ the linear space of all polynomials with coefficients in $\mathbb{C}$.

Theorem 2.1. Let $E \in \mathcal{H} B, E(0)=1$, and $E(t) \neq 0$ for $t \in \mathbb{R}$. Assume that $E$ is of minimal exponential type but not a polynomial. Denote by $\left(w_{n}\right)_{n \in \mathbb{N}}$ the (necessarily infinite) sequence of zeros of $E$ counted according to their multiplicities. In the conditions below we choose the value of $\arg w_{n}$ always in $(-\pi, 0)$. If either $(A)$ holds or both $\left(B_{1}\right)$ and $\left(B_{2}\right)$ hold, where

$$
\sum_{n \in \mathbb{N}}\left(\arg w_{n}+\frac{\pi}{2}\right)^{2}<\infty,
$$

$\left(B_{1}\right)$ for all $w \in \mathbb{C}$ we have $\operatorname{Ord}_{w} E=\operatorname{Ord}_{-\bar{w}} E$,

$$
\sum_{\substack{n \in \mathbb{N} \\ \arg }}\left(\arg w_{n}+\frac{\pi}{4}\right)^{2}<\infty,
$$

then $\mathbb{C}[z]$ is a dense linear subspace of $\mathcal{H}(E)$ and the chain of subspaces of $\mathcal{H}(E)$ is of the form

$$
\{0\} \subset \operatorname{span}\{1\} \subset \operatorname{span}\{1, z\} \subset \operatorname{span}\left\{1, z, z^{2}\right\} \subset \cdots \subset \overline{\mathbb{C}[z]}=\mathcal{H}(E) .
$$

Before we come to the proof of this theorem, let us make a couple of remarks.

Remark 2.2. (i) The condition $\left(B_{1}\right)$ can be viewed as a symmetry condition, for it could be replaced by the requirement that $E$ satisfies the functional equation $E^{\#}(z)=E(-z)$.

(ii) If $E \in \mathcal{H} B$ is a polynomial, then $\mathcal{H}(E)=\{p \in \mathbb{C}[z]: \operatorname{deg} p<\operatorname{deg} E\}$ and the chain of subspaces of $\mathcal{H}(E)$ is just the finite chain $\{0\} \subset \operatorname{span}\{1\} \subset \cdots \subset$ $\operatorname{span}\left\{1, \ldots, z^{\operatorname{deg} E-1}\right\}=\mathcal{H}(E)$.

Remark 2.3. In [3] (see also [6] where a similar result is stated in a somewhat different notation) Hermite-Biehler functions of minimal type are considered that satisfy the symmetry condition $E^{\#}(z)=E(-z)$. For such functions it is proved there that the conclusion of Theorem 2.1 follows if one assumes:

$\left(B_{2}^{\prime}\right)$ All but finitely many zeros of $E$ fall in the angle $\left\{w: \arg w \in\left(-\frac{3 \pi}{4},-\frac{\pi}{4}\right)\right\}$.

Obviously, this result is included in the above Theorem 2.1. Moreover, we use a different, more elementary, method of proof than the one used in [3] or [6]. This made it possible to replace the condition $\left(B_{2}^{\prime}\right)$ by $\left(B_{2}\right)$ and to establish a result in the nonsymmetric case.

The proof of Theorem 2.1 depends on some elementary estimates of canonical products, which we shall state as separate lemmata.

Lemma 2.4. Let $\left(w_{n}\right)_{n \in \mathbb{N}}$ be a sequence of complex numbers contained in the lower half-plane $\mathbb{C}^{-}$that has no finite limit point and that satisfies

$$
\sum_{n \in \mathbb{N}} \frac{1}{\left|w_{n}\right|}<\infty .
$$

If we assume that

$$
\sum_{n \in \mathbb{N}}\left(\arg w_{n}+\frac{\pi}{2}\right)^{2}<\infty
$$


then there exists a positive constant $\delta>0$ such that for all subsets $M \subseteq \mathbb{N}$ we have

$$
\inf _{t \in \mathbb{R}}\left|\prod_{n \in M}\left(1-\frac{t}{w_{n}}\right)\right| \geq \delta \text {. }
$$

Proof. Let $w \in \mathbb{C}^{-}$be given. We estimate the distance of $w^{-1} t$ from the point 1 for $t \in \mathbb{R}$ :

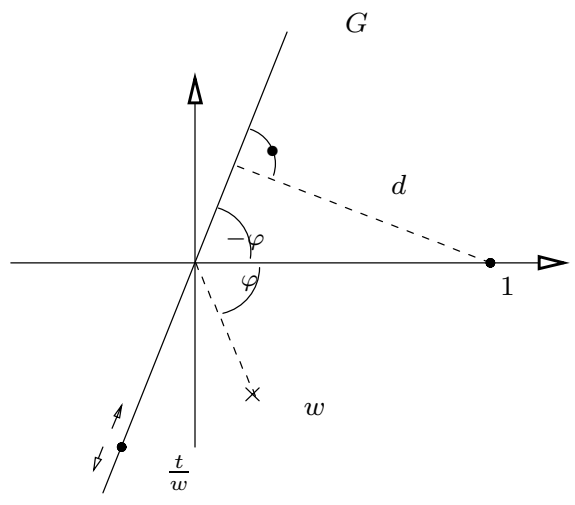

The minimal distance $d$ of the point 1 from the line $G$ is $\sin (-\arg w)$.

Due to the assumption (2.3) every product of the form $P_{M}(z)=\prod_{n \in M}\left(1-\frac{z}{w_{n}}\right)$ is locally uniformly convergent on $\mathbb{C}$. We can estimate $P_{M}$ along the real axis from below:

$$
\left|P_{M}(t)\right|=\prod_{n \in M}\left|1-\frac{t}{w_{n}}\right| \geq \prod_{n \in M} \sin \left(-\arg w_{n}\right) \geq \prod_{n \in \mathbb{N}} \sin \left(-\arg w_{n}\right)=: \delta .
$$

The last product is convergent to some number $\delta \in[0,1]$ since every factor belongs to $(0,1]$. We have to show that $\delta>0$. Taking logarithms shows that this just means that $-\sum_{n \in \mathbb{N}} \log \sin \left(-\arg w_{n}\right)<\infty$. Since $\log x \sim x-1$ for $x \rightarrow 1$, this is equivalent to

$$
\sum_{n \in \mathbb{N}}\left(1+\sin \left(\arg w_{n}\right)\right)<\infty
$$

and by $1+\sin x \sim \frac{1}{2}\left(x+\frac{\pi}{2}\right)^{2}$ for $x \rightarrow-\frac{\pi}{2}$ it is in turn equivalent to (2.4).

Lemma 2.5. Let $\left(w_{n}\right)_{n \in \mathbb{N}}$ be a sequence of points with $\arg w_{n} \in\left[-\frac{\pi}{2}, 0\right)$ that has no finite limit point and satisfies (2.3). If

$$
\sum_{\substack{n \in \mathbb{N} \\ \arg w_{n} \in\left(-\frac{\pi}{4}, 0\right)}}\left(\arg w_{n}+\frac{\pi}{4}\right)^{2}<\infty,
$$

then there exists $\delta>0$ such that for all subsets $M \subseteq \mathbb{N}$,

$$
\inf _{t \in \mathbb{R}}\left|\prod_{\substack{n \in M \\ \arg w_{n}=-\frac{\pi}{2}}}\left(1-\frac{t}{w_{n}}\right) \cdot \prod_{\substack{n \in M \\ \arg w_{n} \in\left(-\frac{\pi}{2}, 0\right)}}\left(1-\frac{t}{w_{n}}\right)\left(1+\frac{t}{\overline{w_{n}}}\right)\right| \geq \delta .
$$

Proof. We again use some elementary estimates of the single factors in the product above. First note that for each $w \in i \mathbb{R}$ and $t \in \mathbb{R}$,

$$
\left|1-\frac{t}{w}\right| \geq \Re\left(1-\frac{t}{w}\right)=1 .
$$


Consider a factor of the form $\left(1-\frac{t}{w}\right)\left(1+\frac{t}{w}\right)$ where $\arg w \in\left(-\frac{\pi}{2}, 0\right)$ and $t \in \mathbb{R}$. We have

$$
\left|\left(1-\frac{t}{w}\right)\left(1+\frac{t}{\bar{w}}\right)\right|=\left|1-\frac{t}{w}\right| \cdot\left|1+\frac{t}{\bar{w}}\right|=\left|1-\frac{t}{w}\right| \cdot\left|1+\frac{t}{w}\right|=\left|1-\frac{t^{2}}{w^{2}}\right| .
$$

If $\arg w \in\left(-\frac{\pi}{2},-\frac{\pi}{4}\right]$, then $\Re w^{-2} \leq 0$ and thus

$$
\left|1-\frac{t^{2}}{w^{2}}\right| \geq \Re\left(1-\frac{t^{2}}{w^{2}}\right)=1-t^{2} \Re \frac{1}{w^{2}} \geq 1 .
$$

Finally, assume that $\arg w \in\left(-\frac{\pi}{4}, 0\right)$ :

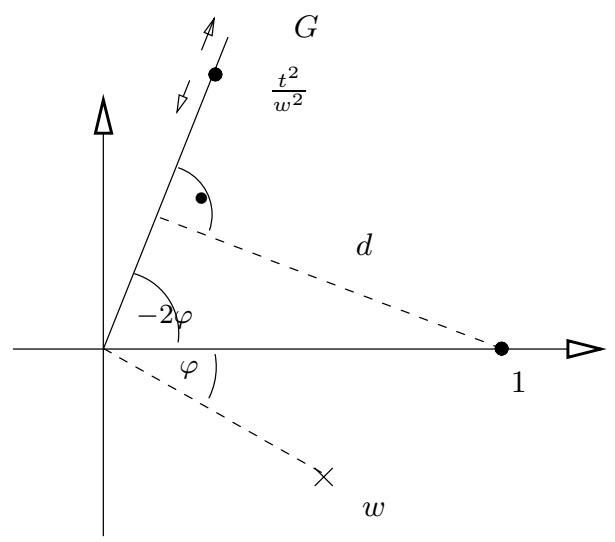

The minimal distance $d$ of the point 1 and the half line $G$ is $\sin (-2 \arg w)$.

Due to the assumption (2.3) the product $Q_{M}(z)$ occurring in the conclusion of the present lemma is locally uniformly convergent on $\mathbb{C}$. Putting together the above estimates of the single factors, we obtain for $t \in \mathbb{R}$,

$$
\left|Q_{M}(t)\right| \geq \prod_{\substack{n \in M \\ \arg w_{n} \in\left(-\frac{\pi}{4}, 0\right)}} \sin \left(-2 \arg w_{n}\right) \geq \prod_{\substack{n \in \mathbb{N} \\ \arg w_{n} \in\left(-\frac{\pi}{4}, 0\right)}} \sin \left(-2 \arg w_{n}\right)=: \delta .
$$

We deduce from the assumption (2.5) that $\delta>0$. We have $\delta>0$ if and only if

$$
\sum_{\substack{n \in \mathbb{N} \\ \arg w_{n} \in\left(-\frac{\pi}{4}, 0\right)}}-\log \sin \left(-2 \arg w_{n}\right)<\infty,
$$

and thus if and only if

$$
\sum_{\substack{n \in \mathbb{N} \\ \arg w_{n} \in\left(-\frac{\pi}{4}, 0\right)}}\left(1+\sin \left(2 \arg w_{n}\right)\right)<\infty .
$$

Since $1+\sin (2 x) \sim 2\left(x+\frac{\pi}{4}\right)^{2}$ for $x \rightarrow-\frac{\pi}{4}$, this condition is equivalent to (2.5).

Let us proceed to the proof of Theorem 2.1. Assume that $E \in \mathcal{H} B$ is subject to the hypothesis of Theorem 2.1 Then, by $(A)$ or $\left(B_{2}\right)$, respectively, the zeros of $E$ are contained in some angle $\{w: \arg w \in[-\pi+\epsilon,-\epsilon]\}$ off the real axis. Thus the condition (2.1) is satisfied. Since $E$ is of minimal exponential type, has no real zeros and is normalized by $E(0)=1$, the representation (2.2) takes the form

$$
E(z)=\prod_{n \in \mathbb{N}}\left(1-\frac{z}{w_{n}}\right) .
$$


We define a sequence $E_{r}, r \in \mathbb{N}$, by recursively removing zeros (or pairs of zeros, respectively) of $E$. Put $E_{1}(z):=E(z)$. If for some $r \in \mathbb{N}$ the function $E_{r}$ is already defined, we pick a zero $w$ of $E_{r}$ that is of minimal modulus among all zeros of $E_{r}$ and define

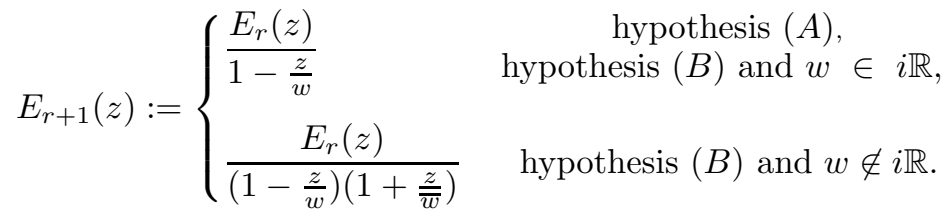

Note that the quotient $p_{r}(z):=E_{r}(z)^{-1} E(z)$ is a polynomial of degree at least $r-1$.

Applying the appropriate one of the Lemmata 2.4 and 2.5 to the sequence $\left(w_{n}\right)_{n \in \mathbb{N}}$ of zeros of $E$ will give a constant $\delta>0$ such that for all $z \in \mathbb{R}$ the estimate

$$
\left|\frac{1}{E_{r}(z)}\right| \leq \frac{1}{\delta}, r \in \mathbb{N},
$$

holds. From the representation (2.6) of $E$, we see that $E$ belongs to the Polya-class, that is, that for each $x \in \mathbb{R}$ the value $|E(x+i y)|$ is a nondecreasing function of $y \geq 0$. By the same argument we conclude that every function $E_{r}$ is of Polya-class. Hence the estimate (2.7) holds not only for $z \in \mathbb{R}$, but throughout $\mathbb{C}^{+} \cup \mathbb{R}$.

We conclude from $E_{r}(z)^{-1}=E(z)^{-1} p_{r}(z)$ that there exist polynomials $p(z)$ of arbitrarily high degree without zeros in $\mathbb{C}^{+} \cup \mathbb{R}$ such that $E(z)^{-1} p(z) \in H^{\infty}\left(\mathbb{C}^{+}\right)$. Since, for $w \in \mathbb{C}^{-}$, with a function $f(z)$ also $(z-w)^{-1} f(z)$ belongs to $H^{\infty}\left(\mathbb{C}^{+}\right)$, this implies that $E(z)^{-1} p(z) \in H^{\infty}\left(\mathbb{C}^{+}\right)$for every polynomial $p$. This in turn shows that $E(z)^{-1} q(z) \in H^{2}\left(\mathbb{C}^{+}\right)$for every polynomial $q$, and hence that $\mathbb{C}[z] \subseteq \mathcal{H}(E)$.

If $w$ is a zero of $E$, denote by $m(w)$ its multiplicity. Consider the polynomials $(1 \leq l \leq m(w), r$ sufficiently large)

$$
F_{(w, l), r}(z):=\left(1-\frac{z}{w}\right)^{-l} p_{r}(z)
$$

We have

$$
\frac{F_{(w, l), r}(z)}{E(z)}=\frac{\left(1-\frac{z}{w}\right)^{-l} p_{r}(z)}{E(z)}=\frac{\left(1-\frac{z}{w}\right)^{-l}}{E_{r}(z)},
$$

and hence by (2.7) the norms $\left\|F_{(w, l), r}\right\|_{\mathcal{H}(E)}$ are uniformly bounded with respect to $r$ :

$$
\left\|F_{(w, l), r}\right\|_{\mathcal{H}(E)}=\left\|\frac{\left(1-\frac{t}{w}\right)^{-l}}{E_{r}(t)}\right\|_{L^{2}(\mathbb{R})} \leq \frac{1}{\delta}\left\|\frac{w^{l}}{(w-t)^{l}}\right\|_{L^{2}(\mathbb{R})} .
$$

Thus there exists a weakly convergent subsequence $\left(F_{(w, l), r_{k}}\right)_{k \in \mathbb{N}}$. Put

$$
F_{(w, l)}:=\mathrm{w}-\lim _{k \rightarrow \infty} F_{(w, l), r_{k}} .
$$

Since weak convergence in a reproducing kernel space implies pointwise convergence and $\lim _{h \rightarrow \infty} p_{h}(z)=E(z)$, we obtain

$$
F_{(w, l)}(z)=\left(1-\frac{z}{w}\right)^{-l} E(z),
$$

and hence $\left(1-\frac{z}{w}\right)^{-l} E(z) \in \overline{\mathbb{C}[z]} \subseteq \mathcal{H}(E)$. 
We can now establish the relation $\overline{\mathbb{C}[z]}=\mathcal{H}(E)$ by proving the following lemma.

Lemma 2.6. Let $E \in \mathcal{H} B$. For a zero $w$ of $E$ denote by $m(w)$ its multiplicity. The linear set

$$
\mathcal{L}:=\operatorname{span}\left\{F_{(w, l)}(z):=\frac{E(z)}{\left(1-\frac{z}{w}\right)^{l}}: w \in \mathbb{C}^{-}, E(w)=0,1 \leq l \leq m(w)\right\}
$$

is dense in $\mathcal{H}(E)$.

Proof. Clearly, the above span $\mathcal{L}$ is a subset of $\mathcal{H}(E)$. Assume on the contrary that it is not dense in $\mathcal{H}(E)$ and choose a nonzero function $G \in \mathcal{H}(E)$ that is orthogonal to $\mathcal{L}$.

Denote by $K(\eta, \zeta)$ the reproducing kernel of $\mathcal{H}(E)$. If $w$ is a zero of $E(z)$ we obtain from the explicit form of $K(\eta, \zeta)$ that for any $k \in\{0, \ldots, m(w)-1\}$,

$$
\left.\frac{\partial^{k}}{\partial \bar{\eta}^{k}} K(\eta, z)\right|_{\eta=\bar{w}}
$$

can, considered as a function in the variable $z$, be written as a certain linear combination of the functions $F_{(w, 1)}, \ldots, F_{(w, m(w))}$. Thus we obtain

$$
G^{(k)}(\bar{w})=\left(G,\left.\frac{\partial^{k}}{\partial \bar{\eta}^{k}} K(\eta, .)\right|_{\eta=\bar{w}}\right)_{\mathcal{H}(E)}=0,0 \leq k \leq m(w)-1 .
$$

Since the numbers $\bar{w}$ are exactly the zeros of $E^{\#}$ (including multiplicities), the Blaschke product $E^{-1} E^{\#}$ is a divisor of $E^{-1} G$ in $H^{2}\left(\mathbb{C}^{+}\right)$, i.e. there exists a function $f \in H^{2}\left(\mathbb{C}^{+}\right)$such that

$$
\frac{G(z)}{E(z)}=\frac{E^{\#}(z)}{E(z)} \cdot f(z) \in \frac{E^{\#}}{E} H^{2}\left(\mathbb{C}^{+}\right) .
$$

This contradicts the fact that

$$
\frac{1}{E} \mathcal{H}(E)=H^{2}\left(\mathbb{C}^{+}\right) \ominus \frac{E^{\#}}{E} H^{2}\left(\mathbb{C}^{+}\right) .
$$

All assertions of Theorem 2.1 are now proved.

By having a closer look at the representation (2.2) of a Hermite-Biehler function of finite order, we obtain a more general version of Theorem 2.1

Corollary 2.7. Let $E \in \mathcal{H B}$. Assume that $E$ is of finite order and has infinitely many zeros. If either the condition $(A)$ or the condition $\left(B_{1}\right)+\left(B_{2}\right)$ holds, then there exists an entire function $D$ with $D^{\#}=D$ and a number $a \geq 0$ such that the chain of subspaces of $\mathcal{H}(E)$ is of the form

$$
\begin{gathered}
\{0\} \subset \operatorname{span}\{D(z)\} \subset \operatorname{span}\{D(z), z D(z)\} \subset \cdots \\
\cdots \subset \overline{D(z) \mathbb{C}[z]}=\underbrace{\mathcal{H}\left(e^{i a z} E(z)\right) \subset \cdots \subset \mathcal{H}\left(e^{i b z} E(z)\right) \subset \cdots \subset \mathcal{H}(E)}_{a \geq b \geq 0} .
\end{gathered}
$$

Proof. The assumption $(A)$ or $\left(B_{2}\right)$, respectively, implies that the condition (2.1) is met, and thus that the function $E$ can be represented as in (2.2). By [5, Lemmata $2.4,2.5]$ and the discussion following it, we see that the chain of subspaces of $\mathcal{H}(E)$ 
is obtained from the chain of subspaces of $\mathcal{H}\left(D^{-1} E\right)$ by multiplication with $D$. Moreover, we know from [5, Theorem 2.7,(ii)] that $\left(a:=-\frac{\tau_{E}}{2} \geq 0\right)$

$$
\mathcal{H}\left(e^{i a z} \frac{E(z)}{D(z)}\right) \subseteq \mathcal{H}\left(\frac{E(z)}{D(z)}\right)
$$

isometrically and that the interval $\left[\mathcal{H}\left(e^{i a z} \frac{E(z)}{D(z)}\right), \mathcal{H}\left(\frac{E(z)}{D(z)}\right)\right]$ is of the desired form.

The function $D(z)^{-1} e^{i a z} E(z)$ is of minimal exponential type because we know that its zeros satisfy (2.1); cf. [1, Lemma 2.10.13]. An application of Theorem [2.1] gives the assertion of the corollary.

Remark 2.8. Note that, if the function $E$ in Corollary 2.7 has only finitely many zeros, we just have to substitute the discrete part $\{0\} \subset \cdots \subset \overline{D(z) \mathbb{C}[z]}$ by the finite chain $\{0\} \subset \cdots \subset \operatorname{span}\left\{D(z), \ldots, z^{\operatorname{deg} E-1} D(z)\right\}$. Compare Remark 2.2, (ii).

\section{FunCTIONS With ZEROS IN A STRIP}

In this section we show that examples of de Branges spaces that satisfy the hypotheses of Theorem 2.1 (or Corollary[2.7) are obtained from even entire functions when the location of the zeros is restricted to a strip.

Theorem 3.1. Let $X$ be an even entire function. Assume that

(1) $X$ is of order at most 2 , finite type, and for some $\alpha \in\left(0, \frac{\pi}{2}\right)$,

$$
\limsup _{r \rightarrow \infty} \frac{1}{r^{2}} \log \left|X\left(r e^{i\left(\frac{\pi}{4}+\alpha\right)}\right)\right| \geq \limsup _{r \rightarrow \infty} \frac{1}{r^{2}} \log \left|X\left(r e^{i\left(\frac{\pi}{4}-\alpha\right)}\right)\right| ;
$$

(2) the zeros of $X$ are located in the strip $\left\{z \in \mathbb{C}:|\Im z|<\frac{1}{2}\right\}$ outside of the angles $\left\{z \in \mathbb{C}: \frac{\pi}{4} \leq|\arg z| \leq \frac{3 \pi}{4}\right\}$.

Define $E(z):=X(\sqrt{i z})$. Then $E$ is an entire function of exponential type, belongs to $\mathcal{H} B$ and has no zeros on $\mathbb{R}$. For each $\delta \in\left(0, \frac{\pi}{2}\right)$ only finitely many zeros of $E$ lie outside the angle

$$
\{z \in \mathbb{C}:-\pi+\delta \leq \arg z \leq-\delta\}
$$

If $X$ additionally is of minimal type and satisfies $X=X^{\#}$, then the chain of subspaces of $\mathcal{H}(E)$ is equal to

$$
\{0\} \subset \operatorname{span}\{1\} \subset \operatorname{span}\{1, z\} \subset \operatorname{span}\left\{1, z, z^{2}\right\} \subset \cdots \subset \overline{\mathbb{C}[z]}=\mathcal{H}(E) .
$$

Proof. Since $X$ is even, the function $E$ is well defined and entire. The zeros $\left(w_{n}\right)_{n \in \mathbb{N}}$ of $E$ are obtained from the zeros $\left(z_{n}\right)_{n \in \mathbb{N}}$ of $X$ lying in the right half-plane by

$$
w_{n}=-i z_{n}^{2}
$$

Since $\left|\arg z_{n}\right|<\frac{\pi}{4}$, we see that $E$ has no zeros in the closed upper half-plane. Moreover, we have

$$
\limsup _{r \rightarrow \infty} \frac{1}{r} \log \max _{\phi \in[0,2 \pi)}\left|E\left(r e^{i \phi}\right)\right|=\limsup _{r \rightarrow \infty} \frac{1}{r^{2}} \log \max _{\phi \in[0,2 \pi)}\left|X\left(r e^{i \phi}\right)\right|<\infty,
$$

and

$$
\limsup _{r \rightarrow \infty} \frac{1}{r} \log \left|E\left(r e^{i \phi}\right)\right|=\limsup _{r \rightarrow \infty} \frac{1}{r^{2}} \log \left|X\left(r e^{i\left(\frac{\phi}{2}+\frac{\pi}{4}\right)}\right)\right|
$$


Hence (3.1) shows that for some $\beta \in(0, \pi)$,

$$
\limsup _{r \rightarrow \infty} \frac{1}{r} \log \left|E\left(r e^{i \beta}\right)\right| \geq \limsup _{r \rightarrow \infty} \frac{1}{r} \log \left|E\left(r e^{-i \beta}\right)\right| .
$$

With the aid of [1, 7.8.1] we conclude that $E \in \mathcal{H} B$.

Let $\gamma \in\left(0, \frac{\pi}{4}\right)$. Since the region

$$
\left\{z \in \mathbb{C}: \Re z \geq 0,|\Im z|<\frac{1}{2},|\arg z| \geq \frac{\pi}{4}-\gamma\right\}
$$

is bounded it contains only finitely many zeros of $X$. Hence only finitely many of the points $w_{n}$ lie in the angles $\{z \in \mathbb{C}: \arg z \in[-\pi,-\pi+2 \gamma] \cup[-2 \gamma, 0]\}$.

The additional hypotheses on $X$ mean that $E$ is of minimal exponential type and satisfies $E^{\#}(z)=E(-z)$. Hence, by Theorem 2.1, we conclude that (3.2) holds.

Example 3.2. The formula

$$
\Xi(z):=-\frac{1}{2}\left(z^{2}+\frac{1}{4}\right) \pi^{-\frac{1}{4}-\frac{i z}{2}} \Gamma\left(\frac{1}{4}+\frac{i z}{2}\right) \zeta\left(\frac{1}{2}+i z\right)
$$

defines an entire function of order 1 and not of finite type. It satisfies the symmetry relations

$$
\Xi(-z)=\Xi(z), \Xi^{\#}(z)=\Xi(z) .
$$

Its zeros are all located in the strip $|\operatorname{Im} z|<\frac{1}{2}$. Moreover, there is exactly one zero $z_{0}$ with $0 \leq \operatorname{Re} z_{0} \leq 6 \pi$, and this zero lies on the real axis. In particular, there are no zeros with argument in $\left[-\frac{3 \pi}{4},-\frac{\pi}{4}\right] \cup\left[\frac{\pi}{4}, \frac{3 \pi}{4}\right]$.

The function $E$ given by Theorem 3.1 with $X(z)=\Xi(z)$ is of finite $\lambda$-type for the growth function $\lambda(r)=\sqrt{r} \log r$; that means

$$
\limsup _{r \rightarrow \infty} \frac{1}{\sqrt{r} \log r} \log \max _{\phi \in[0,2 \pi)}\left|E\left(r e^{i \phi}\right)\right|<\infty .
$$

Its growth can be determined explicitly, in fact

$$
\lim _{r \rightarrow \infty} \frac{\log \left|E\left(r e^{i \psi}\right)\right|}{\sqrt{r} \log r}=\frac{1}{4} \cos \left(\frac{\psi}{2}-\frac{\pi}{4}\right) .
$$

All of the above properties of $\Xi$ with the exception of (B.4) are explicitly stated e.g. in 9]. A proof of (3.4) is easily obtained from Stirling's formula and some estimates of the Riemann zeta function:

(3.4) By the symmetry relation $E(-\bar{z})=\overline{E(z)}$ it suffices to consider values $\psi \in\left(-\frac{\pi}{2}, \frac{\pi}{2}\right]$. By the definition $E(z)=\Xi(\sqrt{i z})$, proving (3.4) amounts to showing that

$$
\lim _{r \rightarrow \infty} \frac{\log \left|\Xi\left(\sqrt{r} e^{i\left(\frac{\psi}{2}+\frac{\pi}{4}\right)}\right)\right|}{\sqrt{r} \log r}=\frac{1}{4} \cos \left(\frac{\psi}{2}-\frac{\pi}{4}\right),
$$

for $\psi \in\left(-\frac{\pi}{2}, \frac{\pi}{2}\right]$, or equivalently (keeping in mind that $\Xi$ is real, putting $\phi:=$ $-\frac{\psi}{2}-\frac{\pi}{4}$, and substituting $r^{2}$ for $r$ )

$$
\lim _{r \rightarrow \infty} \frac{\log \left|\Xi\left(r e^{i \phi}\right)\right|}{r \log r}=\frac{1}{2} \cos \left(\phi+\frac{\pi}{2}\right), \phi \in\left[-\frac{\pi}{2}, 0\right),
$$

uniformly on each compact subset of $\left[-\frac{\pi}{2}, 0\right)$. 
By the definition (3.3) of $\Xi(z)$ we have

$$
\begin{gathered}
\log \left|\Xi\left(r e^{i \phi}\right)\right|=\log \left|\frac{1}{2}\left(r^{2} e^{2 i \phi}+\frac{1}{4}\right)\right|-\frac{\log \pi}{4}-r \frac{\log \pi}{2} \cos \left(\phi+\frac{\pi}{2}\right) \\
+\log \left|\Gamma\left(\frac{1}{4}+\frac{r}{2} e^{i\left(\phi+\frac{\pi}{2}\right)}\right)\right|+\log \left|\zeta\left(\frac{1}{2}+r e^{i\left(\phi+\frac{\pi}{2}\right)}\right)\right| .
\end{gathered}
$$

Obviously, in order to prove the limit relation (3.5), the first three summands in (3.6) are immaterial. Also, the last one is immaterial as well, as we now show. Since by [9, 2.12.2],

$$
(s-1) \zeta(s)=O\left(s^{2}\right), \operatorname{Re} s \geq \frac{1}{2},
$$

the function $(s-1) \zeta(s)$ is of bounded type in the half-plane $\operatorname{Re} s>\frac{1}{2}$. Since $\lim _{t \rightarrow+\infty} \zeta(t)=1$, its mean type is equal to 0 . Moreover, it has only finitely many zeros in each angle $\frac{1}{2}+\left\{w: \arg w \in\left(-\frac{\pi}{2}+\delta, \frac{\pi}{2}-\delta\right)\right\}, \delta>0$. Therefore we know from

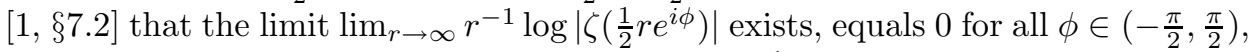
and is attained uniformly in each angular region $\frac{1}{2}+\left\{z \in \mathbb{C}:|\arg z|<\frac{\pi}{2}-\delta\right\}$, $\delta>0$.

An application of Stirling's formula (cf. [8]) will give the desired result: We have, uniformly in each angle $\{z \in \mathbb{C}:|\arg z|<\pi-\delta\}, \delta>0$,

$$
\log \Gamma(z)=\left(z-\frac{1}{2}\right) \log z-z+\frac{1}{2} \log 2 \pi+O\left(\frac{1}{|z|}\right)
$$

Hence,

$$
\begin{gathered}
\log \left|\Gamma\left(\frac{1}{4}+\frac{r}{2} e^{i\left(\phi+\frac{\pi}{2}\right)}\right)\right|=\Re\left[\left(\frac{r}{2} e^{i\left(\phi+\frac{\pi}{2}\right)}-\frac{1}{4}\right) \log \left(\frac{1}{2}\left(\frac{1}{2}+r e^{i\left(\phi+\frac{\pi}{2}\right)}\right)\right)\right] \\
-\left(\frac{1}{4}+\frac{r}{2} \cos \left(\phi+\frac{\pi}{2}\right)\right)+\frac{1}{2} \log 2 \pi+O\left(\frac{1}{r}\right) \\
=\frac{r}{2} \cos \left(\phi+\frac{\pi}{2}\right) \log \left|r+\frac{e^{-i\left(\phi+\frac{\pi}{2}\right)}}{2}\right|-\frac{1}{4} \log \left|r+\frac{e^{-i\left(\phi+\frac{\pi}{2}\right)}}{2}\right| \\
-\frac{r}{2} \sin \left(\phi+\frac{\pi}{2}\right) \operatorname{Arg}\left(\frac{1}{2}+r e^{i\left(\phi+\frac{\pi}{2}\right)}\right)-\frac{r}{2} \cos \left(\phi+\frac{\pi}{2}\right)(1+\log 2) \\
+\frac{1}{4}(\log 2-1)+\frac{1}{2} \log 2 \pi+O\left(\frac{1}{r}\right) .
\end{gathered}
$$

\section{REFERENCES}

1. R. Boas, Entire functions, Academic Press, New York, 1954. MR 16:914f

2. L. de Branges, Hilbert spaces of entire functions, Prentice-Hall, London, 1968. MR 37:4590

3. H.Dym, H.P.McKean, Application of de Branges spaces of integral functions to the prediction of stationary Gaussian processes, Illinois J. 14 (1970), 299-343. MR 41:4642

4. M. Kaltenbäck, H. Woracek, Pontryagin spaces of entire functions I, Integral Equations Operator Theory, 33 (1999), 34-97. MR2000a:46039

5. M. Kaltenbäck, H. Woracek, de Branges spaces of exponential type: General theory of growth, submitted.

6. N.Levinson, H.P.McKean, Weighted trigonometric approximation on $\mathbb{R}^{1}$ with application to the germ field of a stationary gaussian noise, Acta Mathematica (Uppsala) 112 (1964), 99143. MR 29:414

7. M. Rosenblum, J. Rovnyak, Topics in Hardy classes and univalent functions, Birkhäuser Verlag, Basel, 1994. MR97a:30047 
8. E.C. Titchmarsh, The theory of functions, Oxford University Press, Second edition 1939, corrected 1983.

9. E.C. Titchmarsh, The theory of the Riemann Zeta-function, Oxford University Press, 1951. MR 88c:11049

Institut für Analysis und Scientific Computing, Technische Universität Wien, WiedNer Hauptstr. 8-10/101, A-1040 Wien, Austria

E-mail address: michael.kaltenbaeck@tuwien.ac.at

Institut für Analysis und Scientific Computing, Technische Universität Wien, WiedNer Hauptstr. 8-10/101, A-1040 Wien, Austria

E-mail address: harald.woracek@tuwien.ac.at 